\title{
Treatment of vitiligo with autologous cultured keratinocytes in 27 cases
}

\author{
Kyoichi Matsuzaki $\cdot$ Norio Kumagai
}

Received: 8 April 2013 / Accepted: 5 July 2013 /Published online: 28 July 2013

(C) The Author(s) 2013. This article is published with open access at Springerlink.com

\begin{abstract}
Background Vitiligo is an acquired depigmentation of the skin characterized by white spots with well-defined margins, causing psychological stress in patients due to cosmetic concerns. We examined 27 patients who underwent vitiligo treatment using autologous cultured keratinocytes.

Methods The study comprised 20 patients with segmental vitiligo and seven patients with generalized vitiligo, and they were followed up for at least 1 year postoperatively. In all 27 cases, topical steroid or ultraviolet therapy had been previously performed by dermatologists, but this treatment had been ineffective. The patients' vitiligo had stabilized. The patients were treated using keratinocytes obtained from primary culture using Green's techniques or from first passage. Dispase treatment was used to detach the stratified cultured epithelial sheets from their culture dishes. The detached sheets shrank to approximately one half to two thirds of their original size on the culture dish. After the recipient site was completely epithelialized, the skin was exposed to sunlight. Results For patients with segmental vitiligo, 12 had a good therapeutic outcome ( $90 \%$ or more repigmentation) after the first surgery. This number increased to 14 when patients with multiple surgeries were included. There were six patients with fair outcomes (50-90\% repigmentation), and no patients with poor outcomes ( $50 \%$ or less repigmentation). For patients with generalized vitiligo, no patients had a good outcome despite multiple surgeries. There were three
\end{abstract}

\footnotetext{
K. Matsuzaki $(\bowtie)$

Department of Plastic and Reconstructive Surgery, Kawasaki Municipal Tama Hospital, 1-30-37 Shukugawara, Tama-Ku, Kawasaki 214-8525, Japan

e-mail: k4matsu@marianna-u.ac.jp

K. Matsuzaki $\cdot$ N. Kumagai

Department of Plastic and Reconstructive Surgery, St. Marianna

University School of Medicine, Kawasaki, Japan
}

patients with fair outcomes, and four patients with no change outcomes.

Conclusions Cultured keratinocyte grafting was a more effective treatment for segmental vitiligo than for generalized vitiligo. Level of Evidence: Level IV, therapeutic study.

Keywords Cultured keratinocytes · Vitiligo · Phototherapy · Pigmentation $\cdot$ Melanocyte

\section{Introduction}

Vitiligo is an acquired depigmentation of the skin characterized by white spots with well-defined margins, causing psychological stress in patients due to cosmetic concerns. In 1993, we began to treat vitiligo using autologous cultured keratinocytes. In 1997, we treated two patients with segmental vitiligo and two patients with generalized vitiligo and reported no significant difference in the therapeutic outcome [1]. By 2011, a total of 30 vitiligo patients had undergone this treatment and 27 were examined in follow-up. We report here the observed difference in therapeutic outcomes between segmental and generalized vitiligo using autologous cultured keratinocyte therapy.

\section{Material and methods}

Patients

Autologous cultured keratinocytes were used to treat 30 vitiligo patients between February 1993 and March 2011. Among these patients, 20 males and seven females (average age, 24.7 years old) were followed up for at least 1 year postoperatively (average follow-up period, 1.9 years). Segmental vitiligo was present in 20 patients, and seven had generalized vitiligo (Table 1). In all 27 patients, topical 
steroid or ultraviolet therapy had been performed by dermatologists, but the treatments had been ineffective. The patient's vitiligo was stable.

\section{Cultured keratinocytes}

The donor site for keratinocyte harvest was the abdomen or another healthy site selected for its location, i.e., near the graft site or a few centimeters away from the lesion. The method of culturing epithelial cells was the same used in our previous report in which lethally irradiated 3T3-J2 cells and Green's culture techniques were used $[1,2]$. Keratinocytes were obtained from primary culture or from first passage.
Dispase treatment was used to detach stratified cultured epithelial sheets from their culture dishes; the detached sheets shrank to approximately one half to two thirds of their original size on the culture dish and subsequently were attached to carriers. This process enabled the number of melanocytes per unit area to increase in the cultured epithelium before graft transplantation.

Cultured keratinocyte grafting

As in our previous report, the vitiliginous area was deepithelialized using motor-driven, abrasive, serrated wheels. A carrier was used to transfer the cultured epithelium and

Table 1 Treatment of vitiligo

\begin{tabular}{|c|c|c|c|c|c|c|c|c|c|}
\hline $\begin{array}{l}\text { Case } \\
\text { No. }\end{array}$ & Age & Gender & Type & $\begin{array}{l}\text { No. of } \\
\text { operations }\end{array}$ & Donor site & Grafted sites & $\begin{array}{l}\text { Total grafted area } \\
\left(\mathrm{cm}^{2}\right)\end{array}$ & $\begin{array}{l}\text { Follow-up } \\
\text { (year) }\end{array}$ & Result \\
\hline 1 & 9 & M & $\mathrm{S}$ & 1 & Face & Forehead, palpebra & 320 & 6.5 & Good \\
\hline 2 & 9 & M & $\mathrm{S}$ & 2 & Abdomen & Chest, upper limb & 780 & 4 & Good \\
\hline 3 & 40 & $\mathrm{~F}$ & $\mathrm{~S}$ & 1 & Abdomen & Neck, axilla & 600 & 1 & Good \\
\hline 4 & 13 & $\mathrm{~F}$ & $\mathrm{~S}$ & 1 & Abdomen & Forehead, palpebra & 300 & 1 & Good \\
\hline 5 & 38 & M & $\mathrm{S}$ & 1 & Abdomen & Cheek, lip, neck & 480 & 3 & Good \\
\hline 6 & 19 & $\mathrm{~F}$ & $\mathrm{~S}$ & 1 & Face & Cheek, neck & 360 & 1 & Good \\
\hline 7 & 24 & M & $\mathrm{S}$ & 1 & Abdomen & Forehead, palpebra & 300 & 1 & Good \\
\hline 8 & 21 & M & $\mathrm{S}$ & 1 & Face & Face & 180 & 1 & Good \\
\hline 9 & 16 & M & $\mathrm{S}$ & 1 & Face & Face & 480 & 2 & Good \\
\hline 10 & 12 & M & $\mathrm{S}$ & 1 & Face & Palpebra & 30 & 3 & Good \\
\hline 11 & 43 & M & $\mathrm{S}$ & 1 & Inguen & Penis & 60 & 1.5 & Good \\
\hline 12 & 20 & M & $\mathrm{S}$ & 2 & $\begin{array}{l}\text { Upper } \\
\text { thigh }\end{array}$ & Upper \& lower thigh & 1,200 & 4 & Good \\
\hline 13 & 23 & $\mathrm{~F}$ & $\mathrm{~S}$ & 1 & Abdomen & Forehead, palpebra & 140 & 1 & Good \\
\hline 14 & 20 & M & $\mathrm{S}$ & 1 & Abdomen & Scrotum & 180 & 1 & Good \\
\hline 15 & 17 & $\mathrm{~F}$ & $\mathrm{~S}$ & 3 & Abdomen & Abdomen & 2,040 & 3.5 & Fair \\
\hline 16 & 24 & M & $\mathrm{S}$ & 1 & Abdomen & Cheek & 70 & 1 & Fair \\
\hline 17 & 18 & M & $\mathrm{S}$ & 1 & $\begin{array}{l}\text { Upper } \\
\text { thigh }\end{array}$ & Upper thigh & 1,200 & 1.5 & Fair \\
\hline 18 & 38 & $\mathrm{~F}$ & $\mathrm{~S}$ & 2 & Abdomen & Abdomen & 1,620 & 2.5 & Fair \\
\hline 19 & 14 & M & $\mathrm{S}$ & 1 & Abdomen & Face, neck, chest & 900 & 1 & Fair \\
\hline 20 & 22 & M & $\mathrm{S}$ & 1 & Abdomen & Cheek & 215 & 1 & Fair \\
\hline 21 & 29 & M & G & 1 & Abdomen & Neck, chest & 300 & 2.5 & Fair \\
\hline 22 & 22 & M & G & 2 & Abdomen & $\begin{array}{l}\text { Neck, abdomen, lower } \\
\text { thigh }\end{array}$ & 780 & 1 & Fair \\
\hline 23 & 23 & M & G & 3 & Abdomen & Abdomen & 1,320 & 3.5 & Fair \\
\hline 24 & 48 & M & G & 1 & Abdomen & Lip, hand, abdomen & 840 & 1 & $\begin{array}{l}\text { No } \\
\text { change }\end{array}$ \\
\hline 25 & 32 & M & G & 1 & Abdomen & Chin, hand, abdomen & 1,200 & 1 & $\begin{array}{l}\text { No } \\
\text { change }\end{array}$ \\
\hline 26 & 30 & M & G & 1 & Abdomen & Chin, hand, abdomen & 600 & 1 & $\begin{array}{l}\text { No } \\
\text { change }\end{array}$ \\
\hline 27 & 42 & $\mathrm{~F}$ & G & 1 & Abdomen & Neck, hand & 540 & 1 & $\begin{array}{l}\text { No } \\
\text { change }\end{array}$ \\
\hline
\end{tabular}

$M$ male, $F$ female, $S$ segmental type, $G$ generalized type 
grafting was performed [1]. The graft site was dressed with Adaptic $^{\circledR}$ (Johnson \& Johnson Medical Inc., Arlington, TX) cellulose acetate fabric impregnated with petroleum jelly. One week after grafting, the Adaptic ${ }^{\circledR}$ dressing was removed and the site was redressed with steroid ointment-impregnated Adaptic ${ }^{\circledR}$ for 2 to 3 weeks postoperatively.

\section{Management after grafting}

The recipient site was completely epithelialized between 10 and 14 days postoperatively; steroid ointment was applied twice daily after complete epithelialization. The grafted melanocytes were then activated by ultraviolet radiation. Sun exposure commenced 1 week after completion of epithelialization ( 3 weeks postoperatively). The skin was exposed to indirect sunlight, beginning with approximately 5-10 min of treatment per day. Subsequently, the irradiation time was extended to $30 \mathrm{~min}$ to $1 \mathrm{~h}$. The patient was prohibited from receiving prolonged direct sunlight for 1 year after grafting to prevent blistering of the graft site. Repigmentation generally began to occur approximately 3 months postoperatively. Sun exposure treatment was used for at least 6 months. The graft site of each patient was evaluated at their final examination during the follow-up period. A good outcome was defined as $90 \%$ or more repigmentation, a fair outcome as $50-90 \%$, a poor outcome as $50 \%$ or less repigmentation, and no change outcome. For statistical analysis, each condition was converted to a numerical value according to the following scale: no change, 0 ; poor, 1; fair, 2; and good, 3 . After converting to ordinal data, we used the nonparametric Mann-Whitney $U$ test and investigated the difference in treatment effect between segmental and generalized types. The analysis was performed with IBM SPSS statistics 21 (IBM, Armonk, NY, USA).

\section{Results}

Among segmental vitiligo patients, 12 of 20 had a good therapeutic outcome after the first surgery (case 1, Fig. 1). When patients who achieved a good outcome after multiple surgeries were included, this number increased to 14 (case 2, Fig. 2). There were six patients with fair outcomes (case 18,
Fig. 1 Treatment of segmental vitiligo in case 5 . a Before surgery. b Vitiliginous areas were de-epithelialized and the cultured epithelia were grafted. c Three years postoperatively. The outcome was assessed as good a

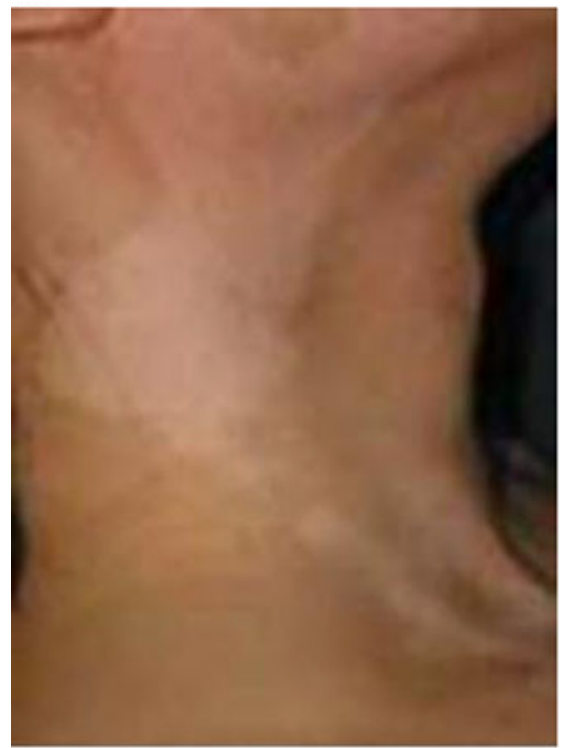

b

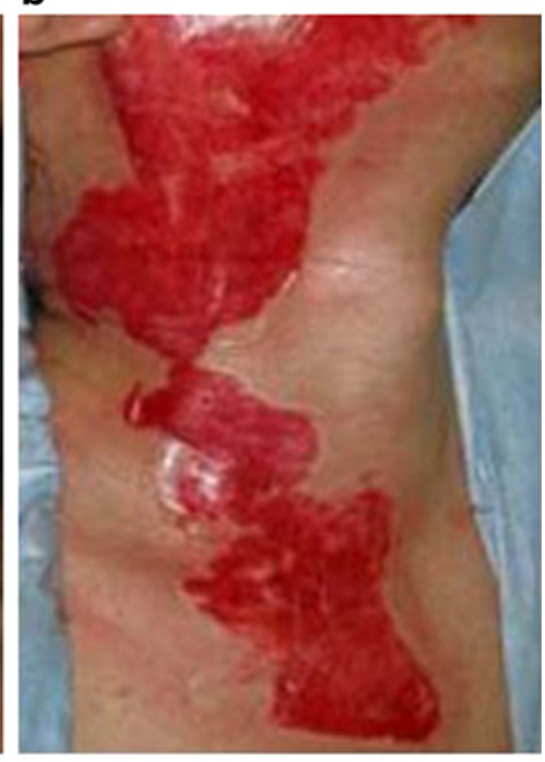

C

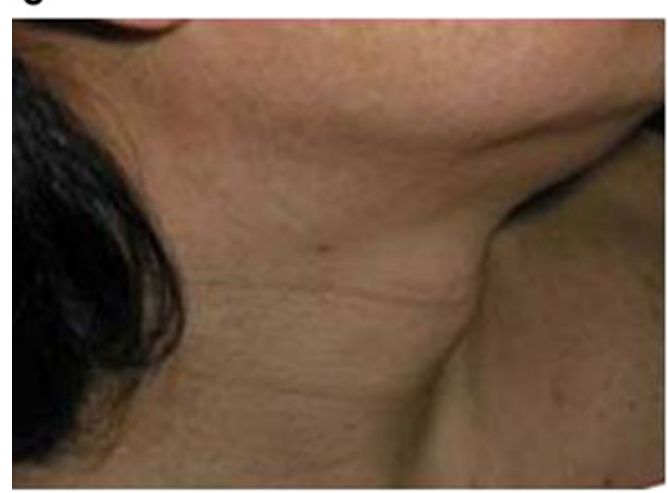


a

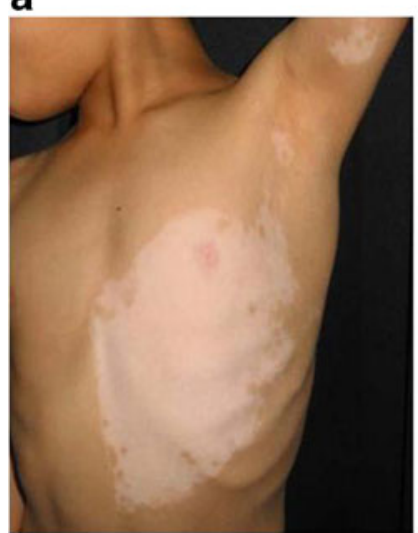

b

Fig. 2 Treatment of segmental vitiligo in case 2. a Before the first surgery. $\mathbf{b}$ Two and a half years after the second surgery. The outcome was assessed as good

Fig. 3), and no patients with poor and no change outcomes. Of the seven generalized vitiligo patients, none had a good outcome despite multiple surgeries. There were three patients with fair outcomes (case 21, Fig. 4), and four patients with no change outcomes (case 24, Fig. 5) (Table 1). We confirmed a highly statistically significant difference in treatment effect between segmental and generalized types $(p=0.000)$. None of the 27 patients experienced a problem related to wound healing, and there was no hypertrophic scarring at the treated sites.

\section{Discussion}

In 1993, we began vitiligo treatment using autologous cultured epithelium [1] after confirming the presence of dopapositive melanocytes with dendritic processes in the basal layer of the epithelium. The desired ratio was approximately one such melanocyte to 14 basal cells. In our previous study, the skin showed histologically improved color at the postoperative follow-up visits. Two weeks after grafting, dopapositive melanocytes survived but melanistic granules were not yet observable. Six weeks postoperatively, there were melanistic granules present, although fewer than are found in normal epithelium. By 6 to 8 months postoperatively, there were dopa-positive melanocytes and melanistic granules at levels similar to those found in normal epithelium. Dopapositive melanocytes were also present in hair follicles; thus, the color of the eyebrow was also improved. At 3 years postoperatively, dopa-positive melanocytes and melanistic granules were arranged in the same pattern as in the normal epithelium. Thus, we demonstrated the effectiveness of autologous cultured keratinocyte treatment and the study was continued with more patients [1].

When the initial two segmental vitiligo cases and two generalized vitiligo cases were evaluated, the treatment appeared to yield similar effectiveness in both types of the disease. However, in our present study of 27 cases, the therapeutic outcomes differed between these types. In some segmental vitiligo cases, such as the patient in case 2 , the outcome was poor after the first surgery but improved to good after the second surgery. There can also be generalized vitiligo cases that can achieve a good outcome after multiple surgeries. Twelve segmental vitiligo cases, but no generalized vitiligo cases, had a good outcome after one surgery. These results indicated a difference in the therapeutic outcome of cultured keratinocyte grafting between the two types of vitiligo.

The autoimmune response is thought to be more virulent in generalized vitiligo cases compared with segmental vitiligo cases [3]. The major cause of the difference in therapeutic outcome might be nonfunctioning melanocytes in the grafted cultured epithelium; these melanocytes having been victimized by the autoimmune response.

The number of melanocytes is known to decrease with repeated passage of the cultured epithelium [4]. Therefore, either a primary culture or a culture obtained after first passage is used for transplantation in vitiligo. If a second passage is performed, a sufficient amount of cultured epithelium can be obtained. Thus, a second passage is frequently used in treatment of extensive burns, but it is unsuitable for vitiligo treatment [5]. Unlike treatment for extensive burns, vitiligo treatment involves a cultured epithelium that has been detached from a culture dish using dispase and that therefore shrinks to approximately one half to two thirds of
Fig. 3 Treatment of segmental vitiligo in case 18 . a Before surgery. $\mathbf{b}$ Two years and 9 months after treatment of the left abdomen. The outcome was assessed as fair a

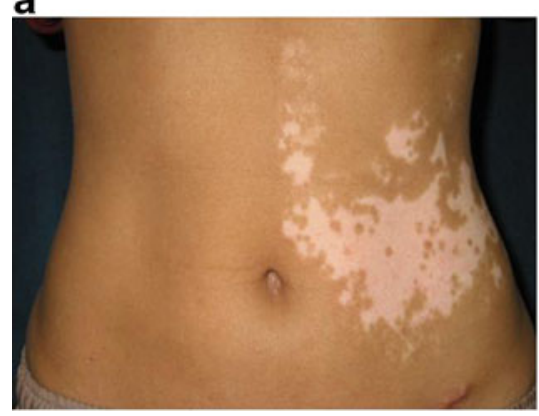

b

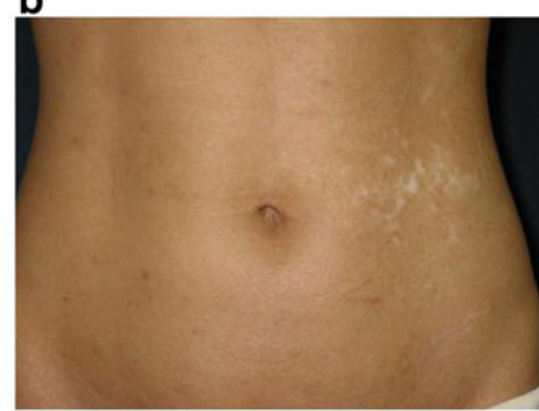


Fig. 4 Treatment of generalized vitiligo in case 21 . a Before surgery. b Two and a half years after treatment of the neck and chest. The outcome was assessed as fair a

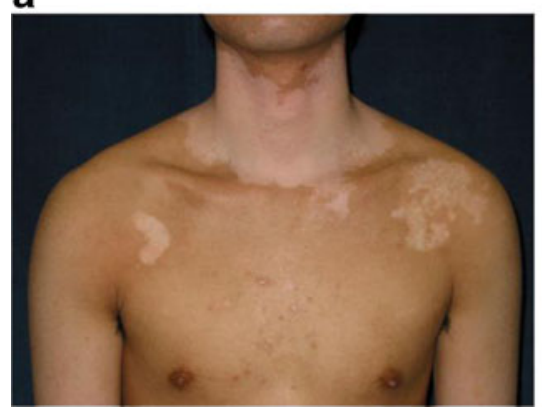

b

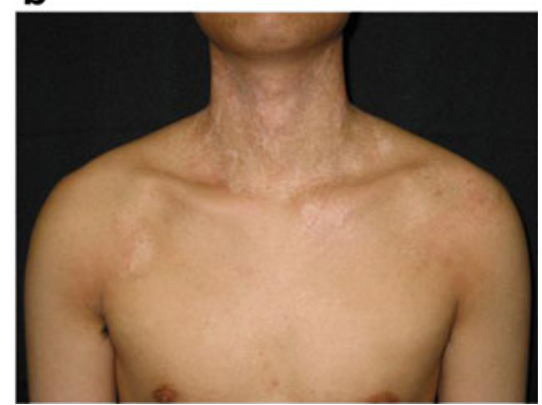

its original size on the culture dish. This process enables the number of melanocytes per unit area to increase before grafting. However, if extensive grafting is required, it is difficult to decide on the acceptable degree of shrinkage. In addition, it is necessary to have a slight overlap when grafting to prevent gap formation between cultured epithelial sheets and to prevent uneven pigmentation. If the grafting site is extensive, the degree of overlap can become insufficient. Cases 2 and 12 were segmental vitiligo patients who had extensive affected areas; they both required a second surgery to achieve a good outcome.

Cultured epithelium is known to maintain characteristics of the donor-site epithelium [6]. Thus, for a palmar graft site, a donor site is required to have a thick horny layer, but without melanocytes; for such a graft, the plantar epithelium of the foot is used. There has not been a paper to date which reported on whether the donor site should be located near the recipient site in the treatment of facial vitiligo. When we first began this particular treatment, we treated facial vitiligo using skin harvested from pre and postauricular regions near the affected site. Since the first goal of vitiligo treatment is to obtain pigmentation, we currently harvest abdominal epithelium, even for grafting to treat facial vitiligo. Non-sun exposed epithelium is known to become well pigmented if it is grafted onto the face [7]. A similar effect is expected for cultured epithelium from the abdomen.

All 27 subjects in our study were Asians. Although Asians are more prone to hypertrophic scarring compared with Caucasians [8, 9], none of our patients developed such complications after treatment. When the vitiliginous area is de-epithelialized to a shallow depth and the cultured epithelium is transplanted, rapid wound healing can be achieved. Thus, we considered this treatment to be safe, even in Asians who are prone to prominent scarring. All our subjects had previously undergone topical steroid or ultraviolet therapy that had proved ineffective with no repigmentation. Thus, all subjects with the therapeutic outcome of fair or better were satisfied with the improvement in color after our treatment.

Felsten et al. [10] reviewed the background and efficacy of different surgical therapies for vitiligo, including blister graft,
Fig. 5 Treatment of generalized vitiligo in case 24 . a Before surgery. b One year after treatment of the upper and lower lips, abdomen, and both hands (not shown). The outcome was assessed as no change a
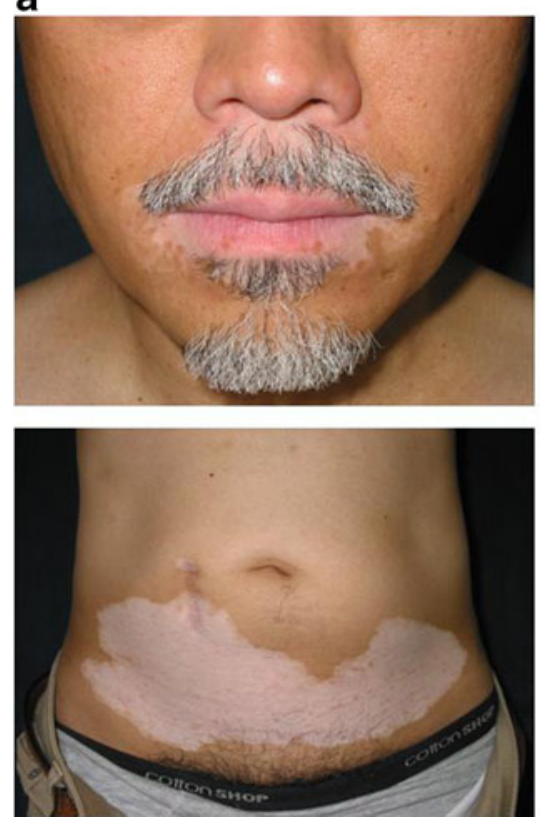

b
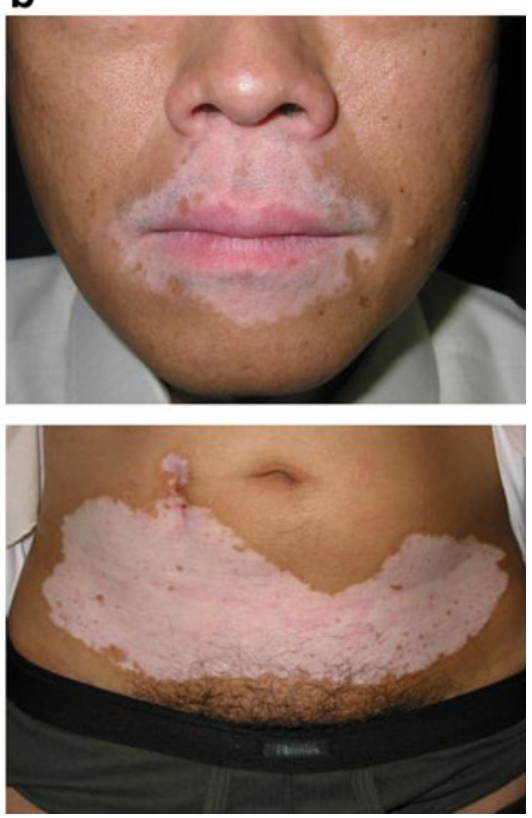
split-thickness skin graft, punch graft, and autologous melanocyte suspension transplant. For transplantation therapies other than those using autologous cultured keratinocytes, many reports have been published. These treatments have included transplantation of cultured autologous melanocytes, keratinocyte/melanocyte cocultures, noncultured suspension of epidermal cells, and noncultured autologous epidermal suspensions produced using a ReCell ${ }^{\circledR}$ system $[11,12]$. Many treatment methods are therefore available for vitiligo, but there has not yet been an optimal treatment identified. Thus, there have been efforts to develop new treatments, such as those using coculture of melanocytes with adipose-derived stem cells to inhibit melanocyte differentiation and to promote melanocyte proliferation and migration [13]. In addition, research on vitiligo treatment has become multifaceted and includes the development of effective carrier dressings for cultured cell delivery to improve therapeutic outcomes [14].

In our previous study, we examined the outcomes of four vitiligo patients who were treated using autologous cultured keratinocytes. We reported no significant difference in the therapeutic outcome between patients with segmental vitiligo and those with generalized vitiligo. In our present study, we examined 27 patients and found that patients with segmental vitiligo had superior therapeutic outcomes compared with those suffering from generalized vitiligo. This study using autologous cultured keratinocytes involved a sufficiently large number of cases to determine an outcome difference. The effectiveness of new treatments can be examined by comparing their results with those of our treatment. Our results might contribute to the development of new treatments for vitiligo.

Acknowledgments The authors are grateful to Dr. Howard Green, George Higginson Professor of Department of Cell Biology at Harvard Medical School, for kindly donating the 3T3. J2 cell line and Dr. Elof Eriksson, Joseph E. Murray Professor of Plastic and Reconstructive Surgery at Harvard Medical School, for reviewing the manuscript and invaluable comments.

\section{Conflict of interest None}

Open Access This article is distributed under the terms of the Creative Commons Attribution License which permits any use, distribution, and reproduction in any medium, provided the original author(s) and the source are credited.

\section{References}

1. Kumagai N, Uchikosi T (1997) Treatment of extensive hypomelanosis with autologous cultured epithelium. Ann Plast Surg 39:6873

2. Rheinwald JG, Green H (1975) Serial cultivation of strains of human epidermal keratinocytes: the formation of keratinizing colonies from single cells. Cell 6:331-343

3. van den Boorn JG, Konijnenberg D, Dellemijn TA, van der Veen JP, Bos JD, Melief CJ, Vyth-Dreese FA, Luiten RM (2009) Autoimmune destruction of skin melanocytes by perilesional $\mathrm{T}$ cells from vitiligo patients. J Invest Dermatol 129:2220-2232

4. De Luca M, Franzi AT, D'Anna F, Zicca A, Albanese E, Bondanza S, Cancedda R (1988) Coculture of human keratinocytes and melanocytes: differentiated melanocytes are physiologically organized in the basal layer of the cultured epithelium. Eur J Cell Biol 46:176180

5. Oshima H, Inoue H, Matsuzaki K, Tanabe M, Kumagai N (2002) Permanent restoration of human skin treated with cultured epithelium grafting: wound healing by stem cell based tissue engineering. Human Cell 15:118-128

6. Kumagai N, Oshima H, Tanabe M, Ishida H, Uchikoshi T (1997) Favorable donor site for epidermal cultivation for the treatment of burn scars with autologous cultured epithelium. Ann Plast Surg 38:506-513

7. Vasconez HC (1994) Skin grafts. In: Cohen M (ed) Mastery of plastic and reconstructive surgery. Little, Brown and Company, Boston, pp 45-55

8. Datubo-Brown DD (1990) Keloids: a review of the literature. Br J Plast Surg 43:70-77

9. Dustan HP (1995) Does keloid pathogenesis hold the key to understanding black/white differences in hypertension severity? Hypertension 26:858-862

10. Felsten LM, Alikhan A, Petronic-Rosic V (2011) Vitiligo: a comprehensive overview Part II: treatment options and approach to treatment. J Am Acad Dermatol 65:493-514

11. Cervelli V, Spallone D, Lucarini L, Palla L, Brinci L, De Angelis B (2010) Treatment of stable vitiligo hands by ReCell ${ }^{\circledR}$ system: a preliminary report. Eur Rev Med Pharmacol Sci 14:691-694

12. Mulekar SV (2005) Long-term follow-up study of 142 patients with vitiligo vulgaris treated by autologous, noncultured melanocyte-keratinocyte cell transplantation. Int $\mathrm{J}$ Dermatol 44:841-845

13. Kim JY, Park CD, Lee JH, Lee CH, Do BR, Lee AY (2012) Coculture of melanocytes with adipose-derived stem cells as a potential substitute for co-culture with keratinocytes. Acta Derm Venereol 92:16-23

14. Eves PC, Bullett NA, Haddow D, Beck AJ, Layton C, Way L, Shard AG, Gawkrodger DJ, Mac Neil S (2008) Simplifying the delivery of melanocytes and keratinocytes for the treatment of vitiligo using a chemically defined carrier dressing. J Invest Dermatol 128:1554-1564 